\title{
Measurement of gas-phase sound speed and thermal diffusivity over a broad pressure range using laser-induced thermal acoustics
}

\author{
Eric B. Cummings and Hans G. Hornung \\ Graduate Aeronautical Laboratories, California Institute of Technology, Pasadena, California 91125 \\ Michael S. Brown and Peter A. DeBarber \\ Applied Spectroscopy Group, MetroLaser, Irvine, California 92714
}

Received March 9, 1995

\begin{abstract}
We report on the detection and analysis of signals generated from gas-phase laser-induced gratings over a large range of static pressure (0.04-100 atm). We employed the experimental technique of laser-induced thermal acoustics and performed measurements on mixtures of $\mathrm{NO}_{2}$ in air and $\mathrm{CO}_{2}$ as a function of pressure. Accurate analysis of the acquired data was obtained from a full theory, including beam size effects. The theory fully reproduces the observed data for a ratio of molecular mean free path to grating wavelength extending from 1 to $4 \times 10^{-4}$. Nonlinear, least-squares fits between modeled and experimental signals provided accurate values of the sound speed and thermal diffusivity.
\end{abstract}

Previously used in optical diagnostics of liquids and solids, ${ }^{1}$ laser-induced gratings have recently been used to measure gas properties at room and elevated temperatures. $^{2-5}$ Measurements have been made at pressures as high as $10 \mathrm{~atm},{ }^{6}$ and two groups have reported theoretical studies of the laser-induced grating signal in gas-phase measurements. ${ }^{3,7}$

In this study we investigated the ability to make and model accurately transient grating measurements over a large range of static pressure. Measurements were made on $\mathrm{NO}_{2}$ buffered in air and $\mathrm{CO}_{2}$ at various concentrations. The experiments were conducted with the previously discussed laser-induced grating technique of laser-induced thermal acoustics ${ }^{2}$ (LITA). When a laser-induced grating is imposed rapidly on a gaseous medium, the medium responds with the generation of a thermal grating and two counterpropagating acoustic waves. Temporal resolution of the light scattered by these structures permits the measurement of the dynamic behavior of the thermal grating and acoustic waves and the extraction of sound speed and transport properties, including the thermal diffusivity. The amplitude and temporal behavior of the induced grating reflect the competing processes of energy deposition, electrostriction, mass and thermal diffusion, and viscous damping.

The experimental arrangement (details in Ref. 2) employed a forward-scattering beam geometry. The output of a YAG-pumped dye laser (5-ns pulse width, $590 \mathrm{~nm}$, and $\geq 10 \mathrm{~mJ} /$ pulse) was divided into two beams, which were spatially and temporally overlapped in a static cell. These two beams were collinearly polarized and formed gratings with wavelengths of approximately $10-30 \mu \mathrm{m}$. The $488-\mathrm{nm}$ output of an $\mathrm{Ar}^{+}$laser was Bragg matched to this grating to generate a signal beam. All three incident beams were focused by a 500-mm lens. The signal beam was time resolved over a period of $500 \mathrm{~ns}$ at low pressure to $40 \mu \mathrm{s}$ at high pressure.

0146-9592/95/141577-03\$6.00/0
We conducted experiments at room temperature by first filling an evacuated static pressure cell with a known amount $\left(\leq 10^{-4} \mathrm{~atm}\right)$ of pure $\mathrm{NO}_{2}$, which resonantly absorbs light from the dye laser. Fixed amounts of dry air or $\mathrm{CO}_{2}$ were then successively added to the cell. The composition was determined by pressure measurements made with Bourdon-type gauges with $0.25 \%$ accuracy. After each introduction of buffer gas, the LITA signal was acquired with a 3-1000 laser shot average as needed for signal-to-noise ratios sufficient to expose any systematic problems with the model. In this way, data were collected as a function of pressure for a constant partial pressure of $\mathrm{NO}_{2}$. Once the highest practical pressure was reached, data were again collected as a function of total pressure as fractional amounts of the mixture were bled off. The grating Knudsen number, the ratio of mean free path to grating wavelength, ranged from near unity at the lowest pressures to $\sim 4 \times 10^{-4}$ at the highest pressure. During an experimental pressure survey it was not necessary to make any changes in beam geometry or to reposition the detector.

The acquired LITA signals were fitted by a previously described theoretical treatment ${ }^{7}$ that involves a solution of the linear hydrodynamic equations with driving terms that are due to absorption followed by collisional energy transfer (thermalization) and electrostriction and includes the effects of finite beam size. The model fits were performed in an iterative process by a nonlinear least-squares algorithm. The model permits the variation of six gas and three experimental parameters: sound speed, thermal diffusivity, mass diffusivity, acoustic damping coefficient, thermalization rate, relative magnitude of thermalization and electrostriction, crossing angle of the beams, and the diameters of the dye laser beams and the $\mathrm{cw}$ laser beam. To reduce the number of independent variables we assumed that the Prandtl (ratio of viscosity to thermal diffusion) and Lewis (ratio of mass 
to thermal diffusion) numbers of the gas were constant with pressure. The beam-crossing angles were measured directly. We estimated beam diameters by noting beam-energy transmission through a calibrated pinhole placed at the beams' focus. These values along with ideal gas law estimates of the other parameters were used as initial guesses in the fitting procedure. Fits were made for each pressure in a given experimental run. The fitted values of the experimental parameters were checked against the measured values for consistency. The crossing angle and beam widths were then held constant, and the fitting was repeated with a succession of small changes in the beam widths until a least-squares minimum was found over the entire pressure range. At this point, the beam widths were held fixed, and fitting was done to determine the sound speed and thermal diffusivity. The thermalization rate and the ratio of thermalization to electrostriction were free parameters during all fitting iterations; however, thermalization completely dominated electrostriction in the $\mathrm{NO}_{2}$ /air measurements, and the thermalization rate was effectively infinite at pressures above a few atmospheres. We note that at $0.55 \mathrm{~atm}$ the value for the fitted thermalization rate amounted to $1 / 4$ that calculated based on a single electronic quenching collision. This suggests the importance of multistep collisions in the thermalization process. The model was able to accurately fit signals acquired from $\mathrm{NO}_{2}$ /air mixtures over a pressure range of $0.04-100 \mathrm{~atm}$.

Examples of LITA signal histories at selected pressures and the corresponding model fits appear in Figs. 1 and 2. The ringing in the signal decays as the acoustic waves propagate away from the overlapping laser beams and are viscously damped. Figure 1 displays experimental and modeled signal histories taken at low pressures. The acoustic gratings decay before they leave the sample volume. Therefore, ringing appears throughout the signal. At the lowest pressures used in this study $(\sim 0.04 \mathrm{~atm})$ the Knudsen number is relatively large $(\sim 1)$ and the spatial coherence of the laser-induced gratings degrades as a result of linear molecular translation. Slightly more than one cycle of the ringing is present. Additionally, the relatively slow collisional rate at which the thermal grating is generated limits the amplitude of the ringing. Electronic quenching of the $\mathrm{NO}_{2}$ molecules $^{8}$ yields a thermalization rate of only $1.6 \times 10^{6} \mathrm{~s}^{-1}$ at $0.036 \mathrm{~atm}$. With increasing pressure the thermalization rate increases and the Knudsen number decreases such that the grating fidelity remains high for increasingly longer periods of time. When the mean free path is small compared with the grating spacing, grating decay is diffusional.

In contrast, Fig. 2 shows a measurement and a model result for $34 \mathrm{~atm}$, where the Knudsen number is quite small, $\mathrm{Kn}=0.0013$. The long tail of the signal is due to Bragg scattering off the thermal grating that persists after the acoustic gratings have propagated away from the sample volume. For pressures that lie between those depicted in Figs. 1 and 2, the LITA signal structure changes smoothly and continuously. For pressures above $34 \mathrm{~atm}$, the length of the thermal tail continues to grow with increasing pressure. The vertical scales in Figs. 1 and 2 are arbitary and not corre-

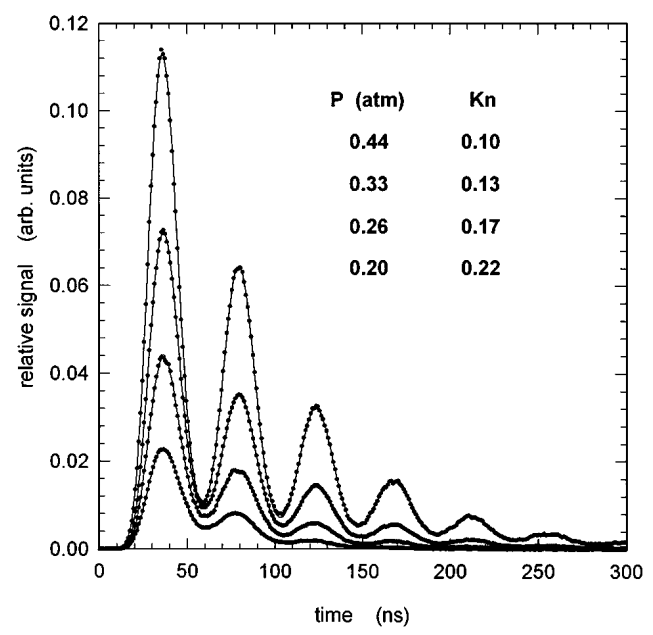

Fig. 1. Measured and theoretical LITA signals from $\mathrm{NO}_{2}$ in air at low pressure. Time zero corresponds to the passage of the grating-inducing beams in the cell. Dots denote experimental data, and the solid curves denote theoretical fits. The curves are nested from bottom to top in order of increasing pressure.

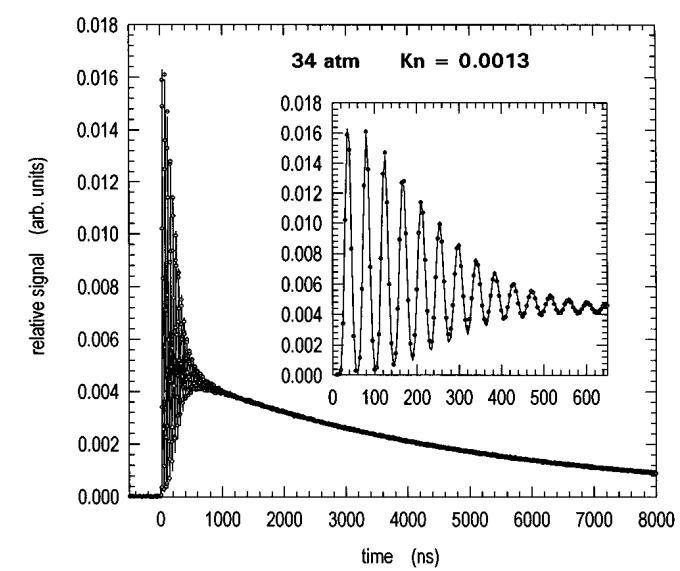

Fig. 2. Measured and theoretical LITA signals from $\mathrm{NO}_{2}$ in air at $34 \mathrm{~atm}$.

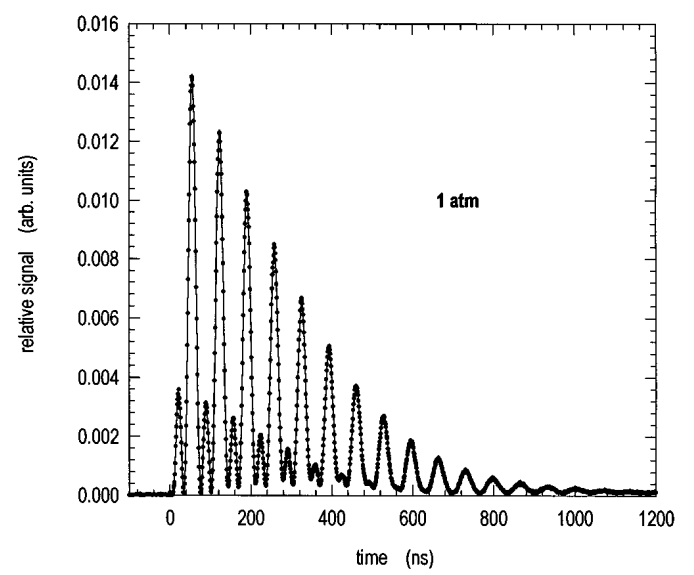

Fig. 3. Measured and theoretical LITA signals from $\mathrm{NO}_{2}$ in $\mathrm{CO}_{2}$ at $1 \mathrm{~atm}$. Both thermal and electrostrictive grating components are present. Every second data point has been plotted. 


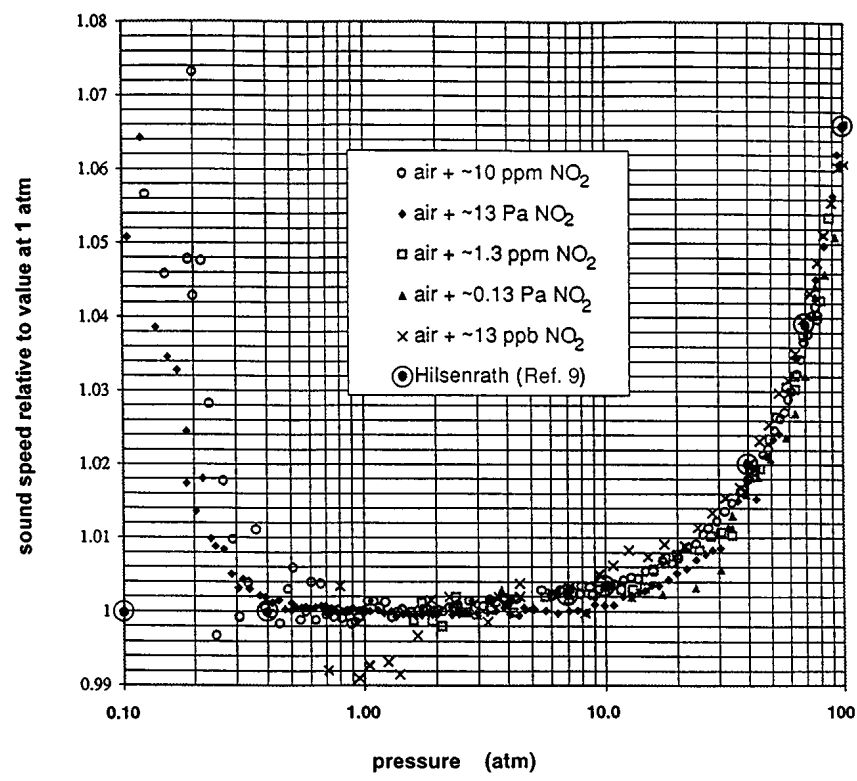

Fig. 4. Sound speed determined from LITA signals as a function of pressure for $\mathrm{NO}_{2} /$ air mixtures.

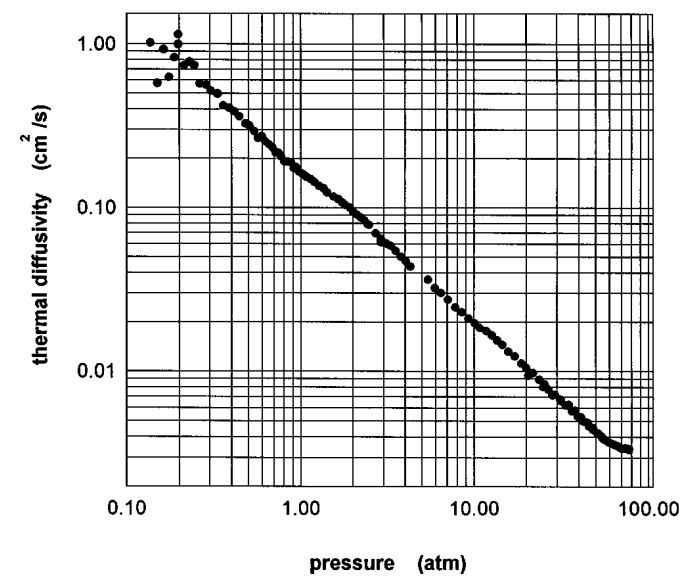

Fig. 5. Thermal diffusivity determined from LITA signals for $\mathrm{NO}_{2}$ in air.

lated with each other. The overall amplitude of the LITA signals increased with increasing pressure because of increasing thermalization rates and decreasing diffusion rates.

The relative contribution of thermal and electrostriction to the laser-induced grating signal depends on various gas and laser beam parameters of a given measurement. For the $\mathrm{NO}_{2}$ /air experiments the thermal grating dominated the electrostrictive grating. By comparison, Fig. 3 shows a measurement made of $\mathrm{NO}_{2}$ in $\mathrm{CO}_{2}$ at $1 \mathrm{~atm}$. Both thermal and electrostrictive grating components are visible in the signal. The electrostrictive component has a frequency twice that of the thermal component.

Figure 4 shows the sound speed extracted from the $\mathrm{NO}_{2}$ /air LITA signals as a function of pressure for a series of fills and drains. The sound speed has been normalized to the value for room temperature and pressure, and values taken from the literature ${ }^{9}$ are included. The relative accuracy of the measurement is better than $1 \%$ for pressures above $1 \mathrm{~atm}$. The systematic increase in the extracted sound speed below
$1 \mathrm{~atm}$ is probably due to the gas mixture's behaving less like a continuous fluid and more like a collection of discrete particles over the distance of the grating spacing. The extracted sound speed approaches the value for the mean thermal velocity, which typically is greater. Despite this systematic deviation, a relative accuracy of better than $3 \%$ remains. Systematic deviations between different runs is evidence of small day-to-day changes in the beam geometry.

Figure 5 shows extracted values for the thermal diffusivity of air. This quantity varies inversely with density. From the values for the thermal diffusivity we determined the thermal conductivity, which is nearly independent of pressure, rising slightly with increasing pressure. Within experimental uncertainty, our inferred value for the thermal conductivity agrees with recent independent measurements ${ }^{10}$ at high pressure. At $1 \mathrm{~atm}$ our experimental value is $\sim 20 \%$ below the published value. This is due to slight misalignment of the incident beams and multistep thermalization rate effects.

In conclusion, we have demonstrated LITA measurements of gas properties over a pressure range of 0.04-100 atm. A theoretical model that includes finite beam-size effects consistently reproduces the data over the full pressure range. From fits of the experimental signals, we determined sound speed measurements accurate to better than $1 \%$ for pressures equal to or greater than $1 \mathrm{~atm}$ and thermal diffusivity measurements accurate to $\sim 20 \%$ at $1 \mathrm{~atm}$ and $<20 \%$ at high pressure.

This research was supported in part by NASA contract NAS3-27017 and U.S. Air Force Office of Scientific Research contract F49620-93-1-0338. We gratefully acknowledge the technical assistance of Jeff Mach and Bonifacio Calayag.

\section{References}

1. H. J. Eichler, P. Günter, and D. W. Pohl, LaserInduced Dynamic Gratings (Springer-Verlag, Berlin, 1986), Chap. 5, p. 123.

2. E. B. Cummings, Opt. Lett. 19, 1361 (1994).

3. P. H. Paul, R. L. Farrow, and P. M. Danehy, J. Opt. Soc. Am. B 12, 384 (1995).

4. D. E. Govoni, J. A. Booze, A. Sinha, and F. F. Crim, Chem. Phys. Lett. 216, 525 (1993).

5. S. Williams, L. A. Rahn, P. H. Paul, J. W. Forsman, and R. N. Zare, Opt. Lett. 19, 1681 (1994).

6. M. S. Brown, P. A. DeBarber, J. S. Maggart, R. W. Pitz, R. L. Farrow, and P. H. Paul, in AIAA 33rd Aerospace Sciences Meeting (American Institute of Aeronautics and Astronautics, Washington, D.C., 1995), paper 950425.

7. E. B. Cummings, I. A. Leyva, and H. G. Hornung, Appl. Opt. 34, 3290 (1995).

8. G. H. Myers, D. M. Silver, and F. Kaufman, J. Chem. Phys. 44, 718 (1966).

9. J. Hilsenrath, Tables of Thermal Properties of Gases, NBS Circular 564 (National Bureau of Standards, Washington, D.C., 1955).

10. N. B. Vargaftik, L. P. Filippov, A. A. Tarzimanov, and E. E. Totskii, Handbook of Thermal Conductivity of Liquids and Gases (CRC, Boca Raton, Fla., 1994), p. 48. 